\title{
Non-Contact Human Gait Identification through IR-UWB Edge Based Monitoring Sensor
}

\author{
Soumya Prakash Rana*, Student Member, IEEE, Maitreyee Dey, Student Member, IEEE, \\ Mohammad Ghavami, Senior Member, IEEE, and Sandra Dudley, Member, IEEE
}

\begin{abstract}
Non-contact sensors are negating the use of wearables or cameras and providing a rewarding and accepting environment to assist in biomedical applications such as, physiological examinations, physiotherapy, home assistance, rehabilitation success determination, compliance and health diagnostics. In this study, physiological parameter identification of human gait has been demonstrated through an edge based sensor and heuristic approach. Impulse radio ultra-wide band (IR-UWB) pulsed Doppler radar has been employed with a focus on human walking patterns. This work extracts an individual's gait trait from associated biomechanical activity and differentiates the lower limb movement patterns from other body areas via a radar transceiver. It is observed that Doppler shifts alone are not reliable to detect human gait because of frequency shifts occurring across the entire body (including, breathing, heartbeat, and arm movements) where movement occurs. Thus, a heuristic spherical trigonometrical approach has been proposed to augment radar principles and short term fourier transformation (STFT) to identify the gait trait precisely. The experiment presented includes data gathering from a number of male and female participants in both ideal and real environments. Subsequently, the proposed gait identification and parameter characterization has been analysed, tested and validated against popularly accepted smartphone applications where the errors are less than $5 \%$.
\end{abstract}

Index Terms-Gait, Impulse Radio Ultra-Wide Band (IRUWB), Edge Based Sensor, Pulsed Radar, Spherical Trigonometry, Doppler Signature, Short Term Fourier Transformation (STFT).

\section{INTRODUCTION}

$\mathbf{H}$ UMAN gait is a complex mechanism [1] where different muscles coordinate to create human locomotion. Motional events are characterized by parameters such as step frequency, step length, stride length, cadence, walking speed, and the total traversed distance within a certain observation time, etc which can identify and analyse motion. The most commonly used technologies for human gait analysis are ultrasound, infrared video, video, floor sensors, wearable sensors, etc. [2]. These technologies involve the measurement, description, and assessment of kinetic and kinematic parameters that define human gait [3]. Gait events and musculoskeletal functions are quantitatively determined and applied in sports, physiotherapy, home assistance, rehabilitation, health diagnostics, and biometric recognition fields [4], [5]. Additionally in some areas human gait analysis is employed to improve athlete performance [6], monitor patient healing progress [7],

Authors are with Biomedical Engineering and Communications (BiMEC) Research Group London South Bank University, 103 Borough Road, London SE1 0AA, United Kingdom help in cases of Parkinson's disease [8], [9], and recognize individuals through their unique walking pattern [10]. Two types of evaluation are performed to define lower limb movement of a person; subjective (carried out by observing the gait pattern in a clinical environment), and objective (carried out by modern techniques and devices). Objective evaluation is a more accurate evaluation and it reduces the error in parameters caused by subjective observation.

Modern gait analysis technologies are classified into three groups; non-wearable sensors (NWS), wearable sensors (WS), and hybrid methods (combination of NWS and WS). NWS systems include image processing employing fixed sensors placed on the ground with surrounding cameras for data collection. Subsequently, the gait parameters are extracted from image or video frames by filtering or segmentation [11], [12]. Other forms of NWS gait analysis examples are conducted with motion capture sensors [13] and force plates [14] in controlled laboratory observation settings. These systems are effective to determine foot pressure, but unable to measure the components of that pressure [15] and can require a large amount of pressure to be applied for activation, thus may be unsuitable for elderly or weaker patients. For instance, Force Sensitive Resistors (FSR) only produce event detection information or contact timing [16], [17], [18] between the leg and ground which is significant characteristic in pathological gait analysis [19], it does not provide kinematic or spatial swing phase information. Also, this type of NWS systems is limited by immobility, price, and operational cost issues. Gait analysis by WS uses sensors placed at the hips, feet, etc. to assess gait characteristic [20]. The widely used WSs are force sensors (FS), accelerometers, gyroscopes, extensometers, goniometers, electromyography, and active markers, etc. Electromechanical device accelerometers are also used to measure the static or dynamic acceleration force on the feet due to the gravity where, gyroscopes are utilized to determine the orientation or angular velocity of the stance or swing leg during gait. Subsequently provides individual spatio-temporal information which can be integrated and synchronized to impart parameters related to trajectory of foot during walking [21]. Some of the researchers have also developed their own algorithm for gait event detection from accelerometer and gyroscope data e.g., adaptive thresholding has been employed to obtain angular velocity from gyroscopes [22] and peak has been detected to identify heel-strike and toe-off from angular velocity signals [23]. Ground Reaction Force (GRF) is another important parameter in gait analysis and determined by FS. It measures the amount of force applied to a human body by the ground during motion. 
Goniometers are used to study the knees, ankles, hips, and metatarsals including the measure of angle between foot and ankle [24]. Both GRF and goniometer sensors are placed in shoe soles for experiments [25]. Muscle force is also used to characterize gait, which is estimated from electromyogram (EMG) signals [26]. Recently, hybrid clinical gait analysis systems such as, CONTEMPLAS [27], Tekscan [28] have been developed where NWS and WS sensors are used. Continuous Wave $(\mathrm{CW})$ radar systems have also been employed to analyse human gait for biological studies and biometric authentication system [29], [30], where time varying Doppler signatures are measured to quantify human gait. The drawbacks of current systems make this field highly engaging and fruitful for further research. NWS systems, although more accurate, need expensive controlled laboratory conditions to isolate from external factors. Clothing can affect gait parameters collected from NWS and WS systems, restricting their success. Image processing based works are limited by participant's clothing, which affects gait parameters detection, and force researchers to work on image segmentation method rather than significant gait parameters. It adds the overhead complexity and reduces the real-time reading opportunities.

\section{A. Contribution}

UWB pulsed radar has been chosen for this study. This scheme has several advantages over other typical narrowband communication systems such as large bandwidth, short pulse, RF levels that are safe to use, and high resolution, making it suitable for biomechanical applications [31]. Short duration UWB pulsed radar is time modulated and can be individualized. These short duration pulses are less sensitive to multiple reflections making the system robust and resistive within multipath environments [32], [33]. The superior penetration properties of UWB signals limit the effect of clothing and other obstacles e.g. walls. It has the capacity to work with low signal to noise ratios (SNR) enabling it to detect moving objects in hostile environments. This paper describes for the first time, the use of pulsed UWB to identify and filter gait patterns from other simultaneous biomechanical activities such as heartrate, breathing, and arm movements. The contributions of the paper are as follows:

- For the first time a three-dimensional spherical trigonometric based theory has been proposed and implemented alongside IR-UWB and STFT to identify parameters which can define a person's gait trait.

- The proposed work has been realized in both ideal (anechoic chamber) and normal environment to demonstrate its efficiency and robustness.

- The resulting gait parameters (walking speed, leg orientation, and traversed distance) have been validated via popular smartphone applications to prove the correctness of the outcomes.

The remaining sections of the article are organised as follows; the laboratory set-up and UWB data acquisition are detailed in Section II! This section focuses on the proposed methodology and provides details on the time-frequency analysis by STFT and related radar principles, with experimental results provided in Section III. Section IV concludes the paper and provides future research directions for this innovative work.

\section{Methods}

A schematic of the proposed study is shown in Figure 1 Here, both ideal (anechoic chamber) and real environments are considered. Initially, the radar is configured and the raw radar scan data are acquired through a radar application program interface (RAPI). Radar module is also configured to retrieve detection information from the environment.

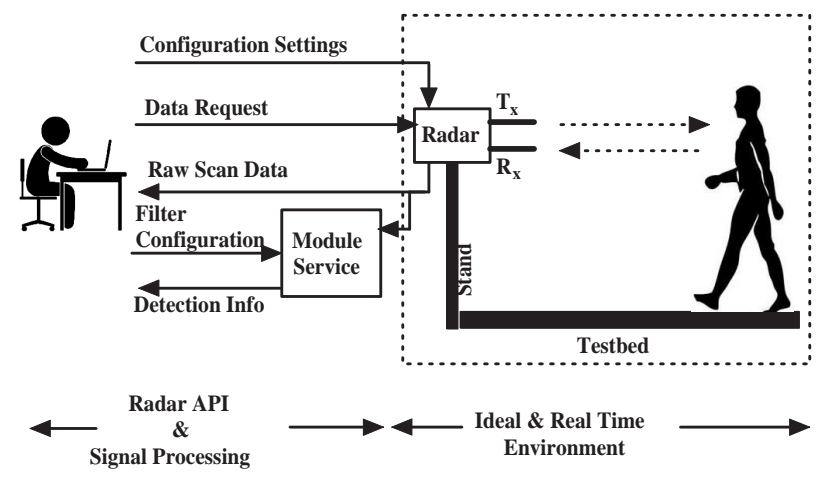

Fig. 1: Schematic diagram of the proposed system.

\section{A. Laboratory Set-up}

A Time Domain PulsON 410 ranging and communications module (P410 RCM) and P410 monostatic radar module (P410 MRM) are used for this and previous work by the team [34], [35], are shown in Figure 3a. The device is a UWB monostatic pulsed Doppler radio transceiver. The architecture utilizes twoway time-of-flight (TW-TOF) range measurement techniques and is used here as hybrid ranging radio and a radar sensor device to non-intrusively measure the human gait. The P410 MRM uses monostatic radar module with omni-directional antennas. The device has been configured before data collection and the same configuration has been maintained for both the chosen (tested) environments. The device configuration has been detailed in Table I] It transmits RF from lower limit of frequency $f_{L}=3.1 \mathrm{GHz}$ to upper limit of frequency $f_{U}=5.3 \mathrm{GHz}$, with the centre frequency at $f_{C}=4.3 \mathrm{GHz}$, and bandwidth of $\left(f_{U}-f_{L}\right)=(5.3-3.1) \mathrm{GHz}=2.2 \mathrm{GHz}$. According to the definition of UWB systems [36], the fractional bandwidth of a device should be more than $50 \%$, in case of P410 device, $\frac{f_{U}-f_{L}}{f_{C}}=\frac{5.3-3.1}{4.3}=51.16 \%$, which follows Federal Communications Commission (FCC) restrictions [37] for power. Transmission power to the antenna port is specified as $-12.64 \mathrm{dBm}$ for safe RF transmission, which abides with FCC regulations [37]. The scan time window for this experiment is 87.84 nanoseconds (ns) long, but the first $5 \mathrm{~ns}$ of the waveform contains noise because of the direct path interference between transmitter and receiver antenna, thus the waveform during the first $5 \mathrm{~ns}$ is filtered out. The scan interval is set to $25000 \mu \mathrm{s}$. The received reflected waveforms are sampled in steps of 61 picoseconds, which results in a 
sampling frequency $f_{s}=16.39 \mathrm{GHz}$, with a Pulse Repetition Interval (PRI) of approximately $100 \mathrm{~ns}$.

TABLE I: Parameter setting of the experiment.

\begin{tabular}{ll}
\hline Parameter & Values \\
\hline Center frequency & \\
Frequency range & $4.3 \mathrm{GHz}$ \\
Scan window & $3.1 \mathrm{GHz}$ to $5.3 \mathrm{GHz}$ \\
Scan interval & 88 nanoseconds \\
Sampling frequency & $25000 \mathrm{microseconds}$ \\
Pulse Repetition Interval (PRI) & $16.39 \mathrm{GHz}$ \\
Transmit gain & 100 nanoseconds (approx) \\
Radar area coverage & $-12.64 \mathrm{dBm}$ \\
Number of antennas & upto 10 meter \\
Ambient operating temperature & one $T_{x}$ and one $R_{x}$ \\
\hline
\end{tabular}

\section{B. Data Acquisition}

Fifteen human participants were engaged in the data collection process. Full ethical approval (Reference Number: Eng_01Dec2017) was gained from London South Bank University, where the research code of practice and ethical guidelines are governed by the university ethics panel (UEP).



Fig. 2: The measured body parts for subjective knowledge collection.

All procedures performed in this study were done so in accordance with the ethical standards of the institutional and/or national research committee and with the 1964 Helsinki declaration and it's later amendments or comparable ethical standards. Initially, gender and anatomical information (height, length of the limbs) have been recorded for each individual as shown in Figure 2. The measured body proportions have been listed in Table III Ideal (in anechoic chamber) and multipath/normal (in laboratory) both the environments have been used for the data collection. These two environments are shown in Figure $3 \mathrm{~b}$ and $3 \mathrm{c}$

\section{Data Processing}

The non-stationary and multi-component radar signal from moving subjects are analysed in this section. All the collected human movement signals have been processed by spherical trigonometry, radar principles, and short term fourier transformation (STFT). A flow chart of this work is provided in Figure 4 Here, the height at which a physiological action takes place has been used to differentiate between lower
TABLE II: Subjective data related to fifteen individuals.

\begin{tabular}{cccccc}
\hline No & Gender & $\begin{array}{c}\text { Height } \\
(m)\end{array}$ & $\begin{array}{c}\text { Leg length } \\
(\mathrm{m})\end{array}$ & $\begin{array}{c}\text { Thigh length } \\
(\mathrm{m})\end{array}$ & $\begin{array}{c}\text { Shank length+ } \\
\text { heel height }(m)\end{array}$ \\
\hline & & & & & \\
1 & Female & 1.58 & 0.85 & 0.43 & 0.42 \\
2 & Female & 1.54 & 0.83 & 0.42 & 0.41 \\
3 & Female & 1.64 & 0.88 & 0.45 & 0.43 \\
4 & Female & 1.73 & 0.93 & 0.47 & 0.46 \\
5 & Female & 1.62 & 0.87 & 0.44 & 0.43 \\
6 & Female & 1.71 & 0.91 & 0.46 & 0.45 \\
7 & Female & 1.69 & 0.91 & 0.46 & 0.44 \\
8 & Male & 1.67 & 0.88 & 0.45 & 0.43 \\
9 & Male & 1.76 & 0.91 & 0.46 & 0.45 \\
10 & Male & 1.71 & 0.88 & 0.45 & 0.43 \\
11 & Male & 1.72 & 0.88 & 0.45 & 0.43 \\
12 & Male & 1.64 & 0.84 & 0.42 & 0.42 \\
13 & Male & 1.78 & 0.92 & 0.47 & 0.45 \\
14 & Male & 1.79 & 0.92 & 0.46 & 0.46 \\
15 & Male & 1.78 & 0.92 & 0.47 & 0.45 \\
\hline
\end{tabular}

and upper body areas. In addition, the orientation of the lower limb has been identified by azimuth or angle of arrival (AoA) calculations. Radar principles have been applied to the pulse waves back-scattered from lower limbs (covers the motion of thigh and shank) to determine the range, walking speed, step length, and total traversed distance. Subsequently, STFT has been performed to derive parameters such as, step frequency and step phase of human gait. The description of the theoretical background has been established and presented in the following subsections.

\section{Azimuth and Elevation Angles}

To assist in the differentiation of body areas, azimuth and elevation angles are considered. Figure $5 \mathrm{a}$ and $5 \mathrm{~b}$ show the elevation angle at a particular time, where $\triangle O A B, \triangle O A B^{\prime}, \triangle O C B$, and $\triangle O C B^{\prime}$ are drawn from the received pulsed radar waveform. Here, $O$ is considered as the radar receiver, which is fixed at a point of height $O P$ from the ground. Therefore, $B C$ and $C B^{\prime}$ represent the height of a moving object from the radar line of sight (LOS) $O A$. The moving body section is elevated from the radar LOS at an angle $\theta$ and below the LOS at an angle $\theta^{\prime}$. Here, $\triangle O A B \cong \triangle O A B^{\prime}$ and $\triangle O C B \cong \triangle O C B^{\prime}$, therefore the height $B C$ and $C B^{\prime}$ can be determined from the trigonometric relationships. Only the calculation of $B C$ from $\triangle O A B$ is explained. Let, the angle between $B C$ and $O B$ be $\alpha$. The travelled distances are $O A, O B$, and $O B^{\prime}$ in propagation delays $t_{1}, t_{2}$ and $t_{2}^{\prime}$ by the pulses, where $t_{1}>t_{2}, t_{1}>t_{2}^{\prime}$ and $O A>O B, O A>O B^{\prime}$. Therefore, the change of distance is $(O A-O B)=\Delta d$, the change of time is $\left(t_{1}-t_{2}\right)=\Delta t$, and speed of light or pulse is $c$. Therefore, pulse can travel the distance in $\Delta t$ is $B C=\Delta \times c$. From the trigonometric ratio in right triangle $\triangle O C B$,

$$
\begin{array}{r}
\cos \alpha=\frac{B C}{O B} \Rightarrow B C=O B \times \cos \alpha \\
\Rightarrow \alpha=\cos ^{-1}\left[\frac{\Delta t \times c}{O B}\right]
\end{array}
$$




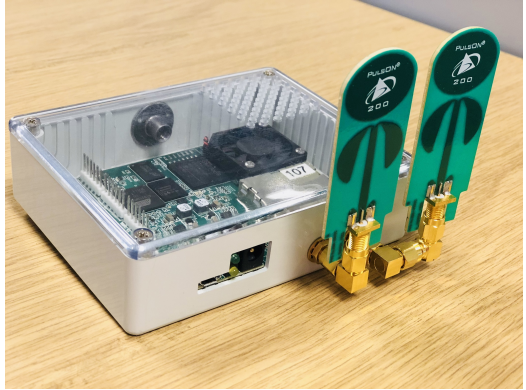

(a) UWB P410 radar module.



(b) Anechoic chamber.

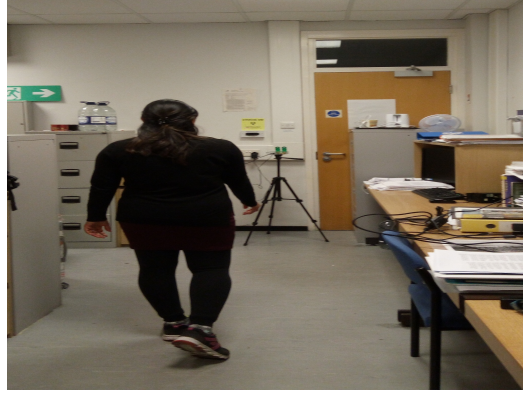

(c) Normal environment.

Fig. 3: UWB device and the environments during data collection.

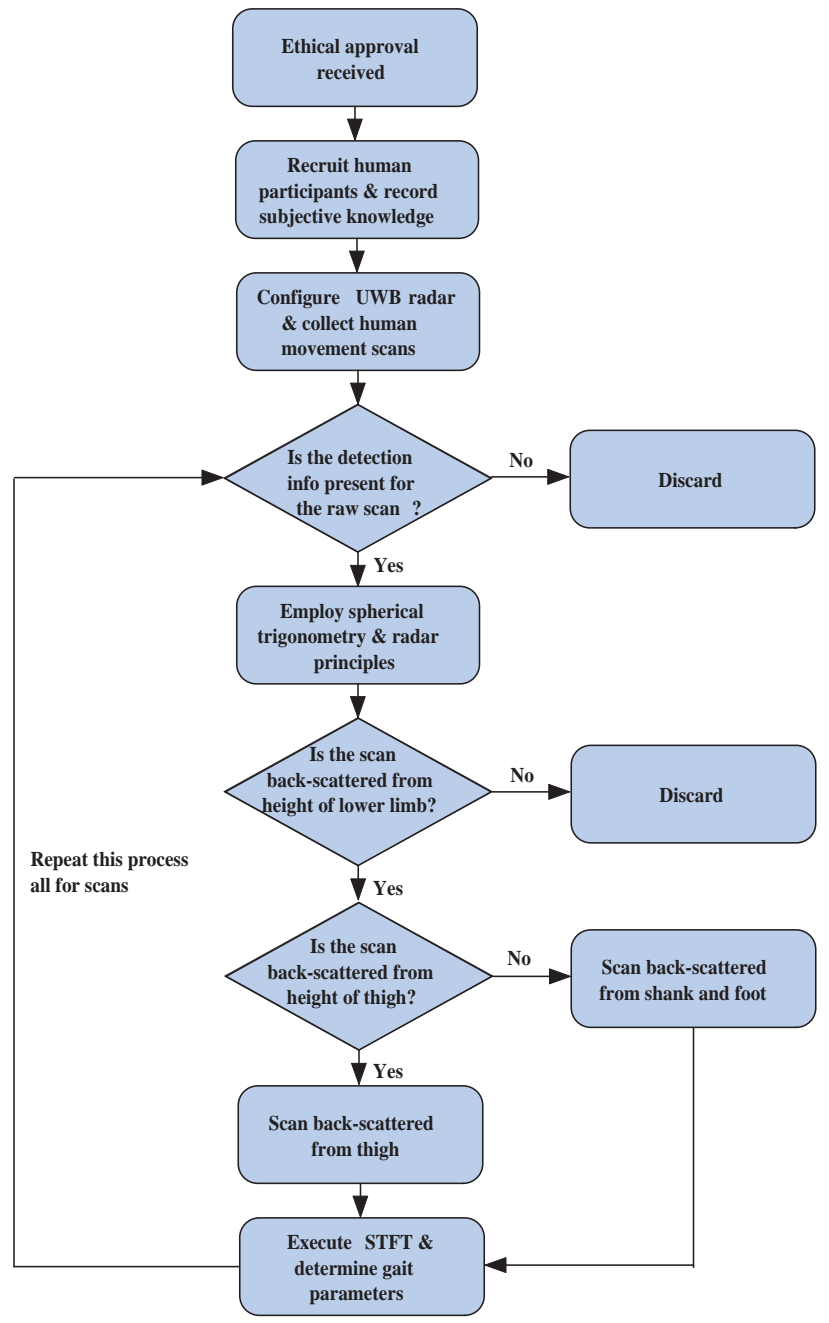

Fig. 4: The flow of processing UWB data and extracting gait information.

Therefore, if the height of a moving object from ground at a particular time is $h$ then,

$$
h=|O P-O B \times \cos \alpha|
$$

This calculation has same outcome when $t_{1}<t_{2}, t_{1}<t_{2}^{\prime}$ and $O A<O B, O A<O B^{\prime}$.



(a) Gait towards radar

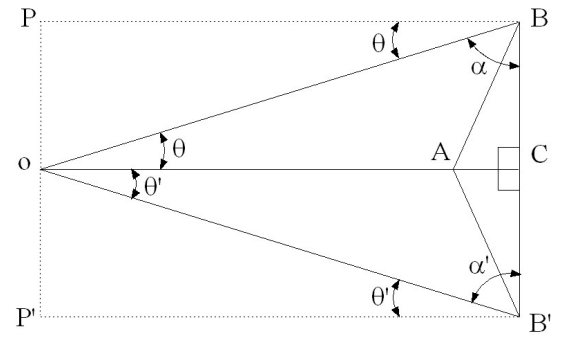

(b) Gait away from radar

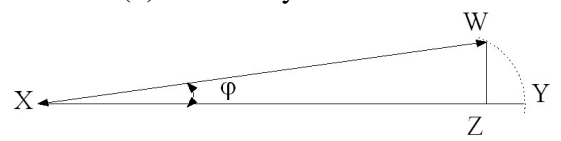

(c) Azimuth during the Gait

Fig. 5: Elevation and azimuth angle during the gait.

Figure 5c displays the calculation of azimuth angle to determine the position or orientation of moving limbs towards the radar. The spherical system measures azimuth angle in a counter clockwise direction from the exact north of the receiver is denoted by $\phi$. Let, the moving limb be deviated at an angle $\phi$, where the travelled distances are $X Y$ and $X W$ in propagation delay $t_{1}, t_{2}$. So, the change of distance is $(X Y-X W)=Y Z$ at the time interval $\left(t_{1}-t_{2}\right)=\Delta t$. The object is deviated from the exact north of the receiver. Now, $Y Z$ is approximately equivalent to the arc $Y W$ created by the object at angle $\phi$. Therefore, $\phi$ is calculated from the radian measure, and equivalent degree conversion is,

$$
\phi=\frac{Y Z \times 360^{\circ}}{X Y \times 2 \times \pi}
$$




\section{E. Range and Velocity}

The height of leg movement has been identified by the developed trigonometric calculations when, the leg length of an individual is known. But, the lower arms can obscure parts of the thigh in motion. Thus, the lower leg (shank) movement has been considered here as a target to determine gait parameters. The target range, $R$ is determined by the round-trip time of the received waveform. Therefore, the range of the moving object is evaluated using $R=\frac{c \triangle T}{2}$ by measuring the time delay where, $c=3 \times 10^{8}$ meter/ seconds is the speed of light, and $\triangle T$ is the propagation delay(s). The velocity of moving object is obtained from the measurement of the Doppler frequency shift with the help of track information which is found from the radar measurements of the target location over a period of time. Thus, the velocity of an object is determined by $f_{d}=\frac{2 v_{p}}{\lambda}$ where, $f_{d}$ is the difference between the frequency of transmitted and received wave or Doppler shift, $v_{p}$ is the radial component of the velocity, and $\lambda$ is the wavelength of the transmitted wave. The range $R$ decreases when the person comes closer to the radar and $f_{d}$ increases and vice versa [38]. Therefore, if the range at $t_{1}$ and $t_{2}$ time are $R_{1}$ and $R_{2}$ respectively then, the change in distance or range is $\left(R_{1}-R_{2}\right)$ which, describes the distance covered by the shank in $\left(t_{1}-t_{2}\right)$ time, and maximum change of distance by the shanks is the step length of a stride. The change in range $\left(R_{1}-R_{2}\right)$ has been summed up each time for shanks to get the total distance covered by an individual.

\section{F. STFT}

As the spectral content of the UWB radar generated waveforms are non-stationary, applying the discrete Fourier transform (DFT) over a long window does not reveal transitions in the movement behaviour. To solve this issue, we can apply the DFT over short periods of time for which the radar signals can be considered as stationary. This can be observed as a time-frequency trade-off. The DFT of the windowed radar waveform is defined as:

$$
\begin{aligned}
S(m, k) & =\left.S(m, \omega)\right|_{\omega=\frac{2 \pi}{N} k} \\
= & \left.\sum_{n=-\infty}^{\infty} s(n) w(n-m) e^{-j \omega n}\right|_{\omega=\frac{2 \pi}{N} k} \\
& =\sum_{n=-\infty}^{\infty} s(n) w(n-m) e^{-j \frac{2 \pi}{N} k n}
\end{aligned}
$$

Where $s(n)$ is the received sequence obtained from the corresponding environment of the experiment sampled at $f_{s}$, $w(n-m)$ is the window function starting at discrete time $m, \omega=2 \pi f$ is the angular frequency with discrete values of $\omega=\frac{2 \pi}{N} k$. Here, we are using the Hamming window length of 40 (experimentally fixed) which is characterised by the following equation:

$$
w(n)=0.54-0.46 \cos \left(\frac{2 \pi n}{N-1}\right)
$$

The discrete STFT [39] is used to create a collection of sequences, each corresponding to the frequency components of $s(n)$ falling within a particular frequency band which corresponds to a certain activity or physiological signal.

\section{G. Signal to Noise Ratio}

The radar takes a period or Scan Training Period (STP) to determine the background noise before any detection occurs. The background noise strength $P_{n o i}$ is measured from the received waveforms during STP. The received signal strength $P_{\text {sig }}$ while detection occurred is determined with the help of radar detection information. The signal to noise ratio (SNR) [38] is defined as the ratio of the power of a received signal and the power of background noise using $S N R=\frac{P_{s i g}}{P_{n o i}}$. Here, the SNR is function of transmitter gain and receiver gain in decibels $(\mathrm{dB})$.

Pseudo-code of the entire process has been provided to demonstrate the execution steps of the proposed study in Algorithm 1] where, the associated theoretical background has been detailed in Section $\amalg-\mathrm{F}$ to $\mathrm{I}-\mathrm{G}$

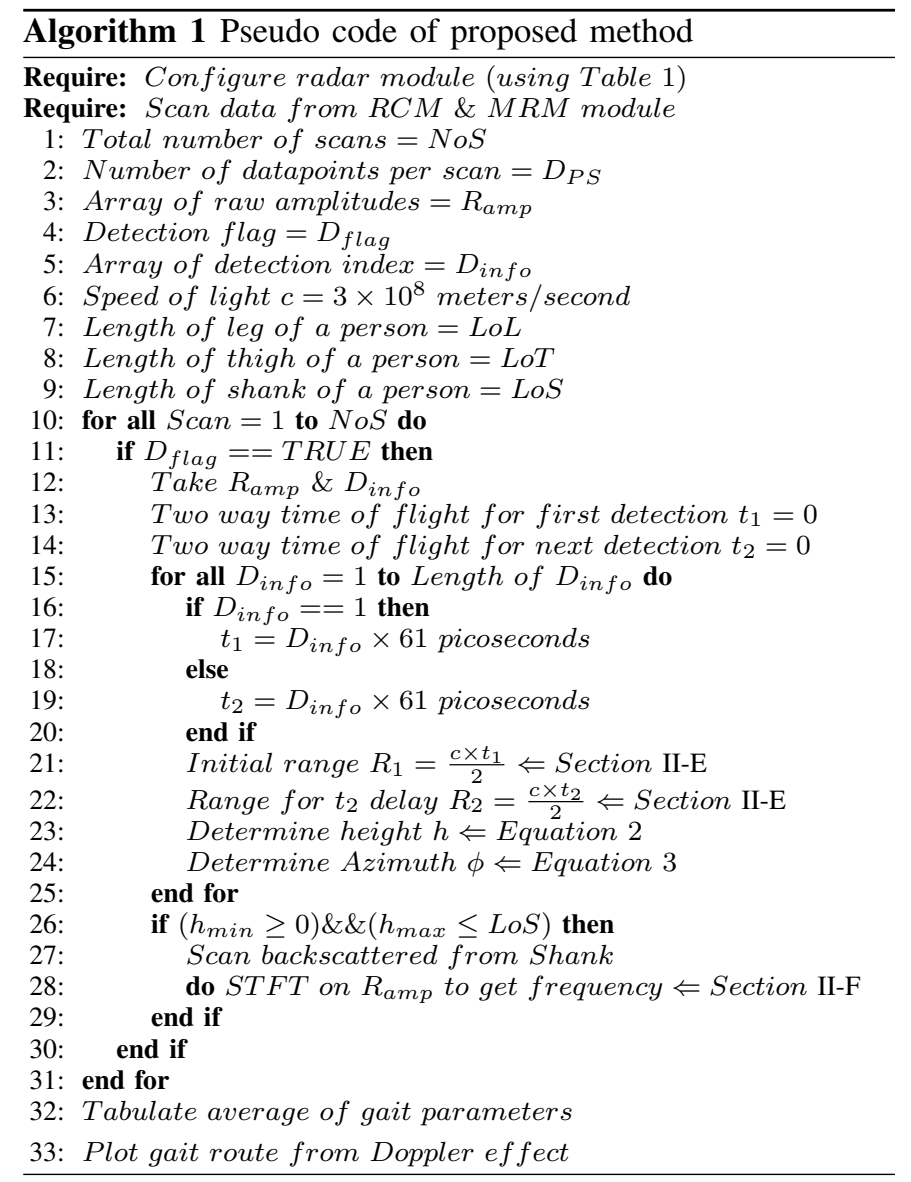

\section{H. Validation}

Three obtained parameters have been validated via popular smartphone applications. The parameters, walking speed, traversed distance during observation period, and lower limb orientation have been compared with the outcomes of accelerometer sensor, Samsung health application, and gyroscope sensor. Each participant has been asked to walk, carrying two smartphones and turning on these applications during their walking phase in both the ideal and normal environment. The accelerometer delivers the linear acceleration, Samsung health application delivers covered distance within fixed time, and the 
gyroscope provides azimuth, pitch, and roll information. The average velocity of an individual has been determined from the average acceleration provided by accelerometer and used for validation purpose against the UWB method. The distance covered within 30 seconds by an individual has been compared with the distance determined by Samsung health application. This work doesn't provide the left and right side information thus, the absolute of azimuth (positive or negative) provided by gyroscope sensor has been employed for validation purpose of azimuths measured by the proposed work.

\section{RESULT ANALYSIS}

Physiological movement data have been accumulated and studied through the proposed spherical trigonometric system (described in Section II-D) which augmenting radar principles (described in Section II-E). Further, the height of the reflected pulses has been determined and subsequently, the recorded anatomical measurements (of Table II) have been used to identify pulsed waveforms back-scattered from different areas of the body. As the focus of this paper is on gait parameter identification the pulses reflected within the height of shank and thigh have been considered for each participant. However, arm swinging and walking are two interrelated physiological events where, arms cover some portion of legs below the waist creating some the redundancy to distinguish between the arm and thigh. Also, the shank reflects the standard osteometrics better than thigh, therefore the movement of shank has solely been considered for characterizing an individual's gait pattern. The pulsed waveforms back-scattered from shank has been transformed by using STFT to determine the frequency of an event that occurs for the movement of shank. The waveforms reflected above the shank and thigh obviously include other physiological actions such as, arm movement, heart rate and breathing patterns and they have been filtered out here. Thus, the height of body section has been considered to discriminate the gait from other bio-mechanical activities. The participants have been asked to walk at different paces (i.e., slow, medium, and fast) in ideal and normal environment both for testing the proposed work and corresponding results have been presented in the following sections.

\section{A. Results from Anechoic Chamber}

The pulses backscatter from the human body when individuals walk back and forth in front of the radar. These pulsed waveforms have been processed in following manner as shown in Figure 4, if a pulsed wave or signal has been identified as reflecting from shank then considered for further processing otherwise it's discarded. The first pulse of each signal reflecting from shank has been transformed by STFT characterizing the step movement of human gait. Figure6 shows the frequency of lower leg movement obtained from the proposed STFT for an observation time of 2 seconds when preferred walking speed of participants are at fast, medium and slow pace. The results for both female and male have been included here. The figure shows the repetitive movement of shanks which demonstrates the step frequency of human gait. Figure $6 \mathrm{a}$ and $6 \mathrm{~b}$ show the step frequency at a fast
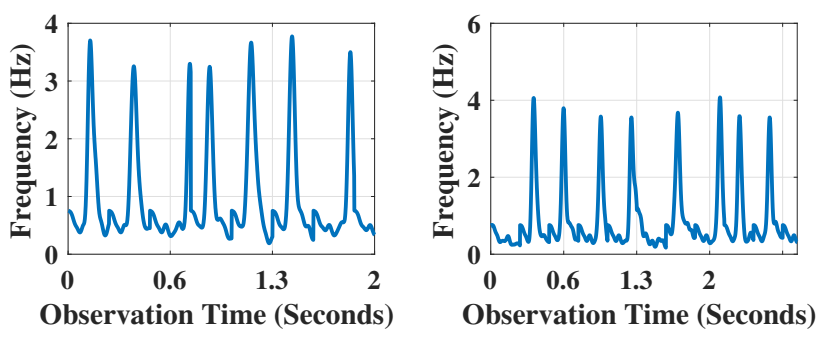

(a) Female walking at fast pace.

(b) Male walking at fast pace.
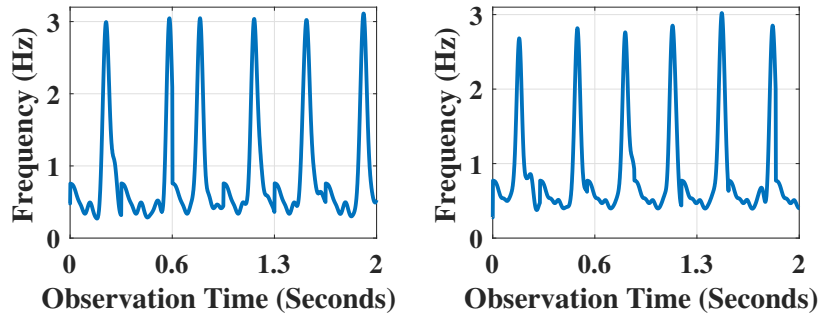

(c) Female walking at medium pace.

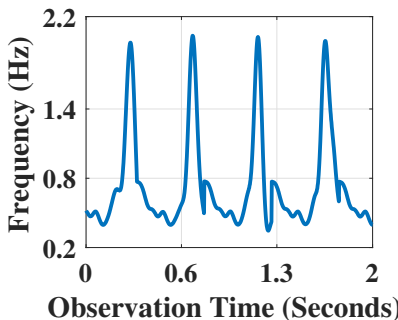

pace.

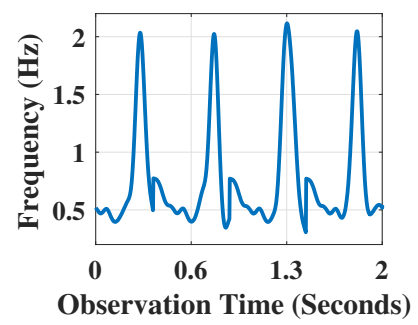

(e) Female walking at slow pace.

(f) Male walking at slow pace.

Fig. 6: Shank movement frequency of participants walking at different pace obtained through UWB radar in ideal environment.

walking pace which reaches up to $3.9 \mathrm{~Hz}$ for females and 4 $\mathrm{Hz}$ for male participants. Figure $6 \mathrm{c}$ and $6 \mathrm{~d}$ displays the shank movement at medium walking pace which results approximately $3 \mathrm{~Hz}$ for both female and male participants. Figure $6 \mathrm{e}$ and 6e demonstrates step frequency of approximately $2 \mathrm{~Hz}$ at slow walking pace for the participants. The variation of step frequency occurs when the participants reach at one end of test bed and walk slowly to turnaround. For example, Figure 6a shows approximately $3.9 \mathrm{~Hz}$ initially but it decreases to 3.2 $\mathrm{Hz}$ when the person reached to the one end of test bed and needed to slow down the walking speed. The step phase or the swing phase also has been determined (1/step frequency) from step frequency and detailed in Table III for all participants.

The relation between SNR and range is plotted in Figure 7 for two female and male participants. The noise figure is evaluated from the STP and SNR is measured from the scans with the help of detection information and logarithmic relation is found between SNR and range.

The route of participant's gait has been determined from Doppler effect. The Doppler shift $f_{d}$ (described in SectionII-E) has been calculated during the observation time and plotted to check the gait route of a person. The identified signals from shanks have been processed through STFT and plotted 


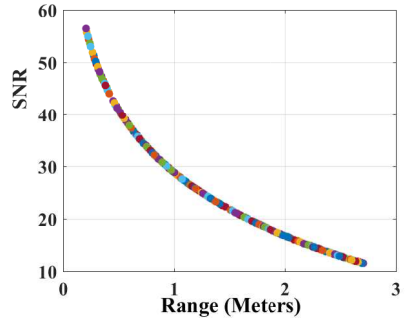

(a) $1^{\text {st }}$ female participant.

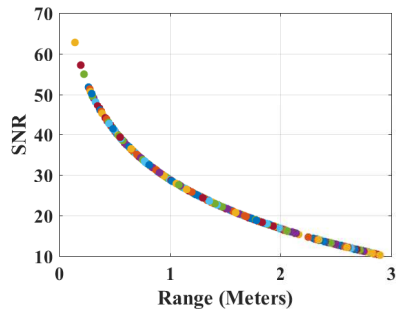

(c) $1^{\text {st }}$ male participant.

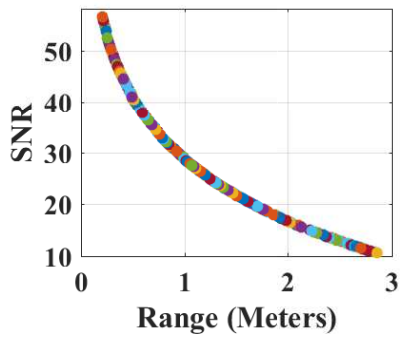

(b) $2^{\text {nd }}$ female participant.

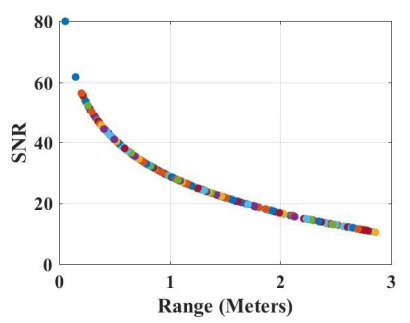

(d) $2^{\text {nd }}$ male participant.
Fig. 7: SNR and range relationship in the ideal environment during movement.

in Figure 8 as received over time. The upside and downside of Figure $8 \mathrm{a}, 8 \mathrm{~b}, 8 \mathrm{c}$ and $8 \mathrm{~d}$ specify the nearest and furthest point from radar during a walk two female and two male participants. Therefore, $\mathrm{x}$-axis represents the increment of time with received signals. The total distance traversed by an individual has been obtained from summing up the changes in range during the locomotion in front of the radar.

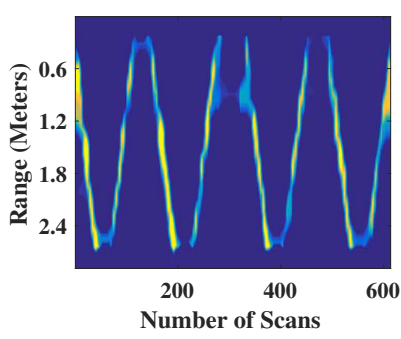

(a) $1^{\text {st }}$ female participant.

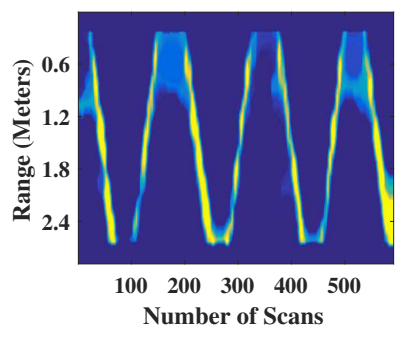

(c) $1^{\text {st }}$ male participant.

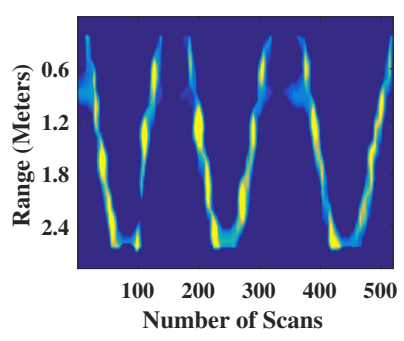

(b) $2^{\text {nd }}$ female participant.

(d) $2^{\text {nd }}$ male participant.

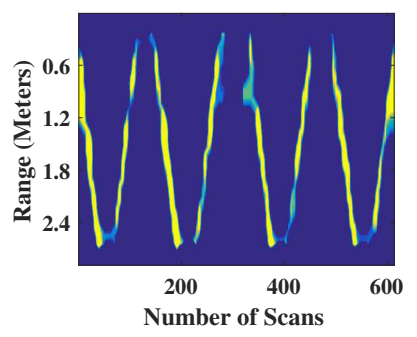

Fig. 8: Movement patterns on test bed in anechoic chamber.

Table III includes the average gait parameters obtained from the experiment for human participants in anechoic chamber/ideal environment. Seven parameters have been calculated from the proposed experimental data to define the human gait. The parameters such as, step phase $\left(S_{p}\right)$, step length $\left(S_{l}\right)$, cadence $\left(C_{a}\right)$, stride length $\left(S T_{l}\right)$, walking speed $\left(W_{s}\right)$, and lower limb orientation $\left(L_{o}\right)$ have been determined along with step frequency $\left(S_{f}\right)$, and total traversed distance $\left(T_{d}\right)$. The step period or step phase has been calculated by estimating period of step frequency (i.e., gait period=1/gait frequency). The maximum change in distance by the shanks describe the step length. Cadence is the number of 'revolutions per minute' (RPM) i.e., the number of steps per minute has been estimated from the gait frequency (i.e., cadence $=$ step frequency $\times 60$ ). Walking speed has been obtained from the frequency shift (described in Section II-E) for each human subject. The angle of arrival (AoA) or azimuths of pulses are calculated from the range and propagation delay $(\mathrm{Eq} 3 \mathrm{3}$ in spherical polar coordinates, providing a clear indication of the orientation of the moving lower limb (shank) with respect to the radar.

TABLE III: Results of gait analysis from ideal environment.

\begin{tabular}{ccccccccc}
\hline No & $\begin{array}{c}S_{f} \\
(\mathrm{~Hz})\end{array}$ & $\begin{array}{c}W_{s} \\
(\mathrm{~m} / \mathrm{s})\end{array}$ & $\begin{array}{c}C_{a} \\
(\mathrm{c} / \mathrm{min})\end{array}$ & $\begin{array}{c}S_{l} \\
(\mathrm{~m})\end{array}$ & $\begin{array}{c}S_{p} \\
(\mathrm{~s})\end{array}$ & $\begin{array}{c}S T_{l} \\
(\mathrm{~m})\end{array}$ & $\begin{array}{c}T_{d} \\
(\mathrm{~m})\end{array}$ & $\begin{array}{c}L_{o} \\
\left({ }^{0}\right)\end{array}$ \\
\hline & & & & & & & & \\
1 & 3.033 & 1.527 & 181.892 & 0.501 & 0.329 & 1.002 & 45.603 & 1.833 \\
2 & 3.050 & 1.522 & 182.783 & 0.498 & 0.328 & 0.997 & 45.608 & 1.714 \\
3 & 3.003 & 1.500 & 180.030 & 0.499 & 0.333 & 0.999 & 45.000 & 1.888 \\
4 & 3.144 & 1.564 & 187.759 & 0.521 & 0.347 & 1.042 & 46.910 & 1.876 \\
5 & 2.954 & 1.469 & 176.398 & 0.489 & 0.326 & 0.979 & 44.072 & 1.762 \\
6 & 3.271 & 1.629 & 195.532 & 0.542 & 0.361 & 1.085 & 48.852 & 1.954 \\
7 & 2.815 & 1.400 & 168.026 & 0.466 & 0.310 & 0.932 & 41.980 & 1.679 \\
8 & 3.018 & 1.499 & 179.986 & 0.499 & 0.333 & 0.999 & 44.968 & 1.798 \\
9 & 2.990 & 1.489 & 178.790 & 0.496 & 0.330 & 0.992 & 44.669 & 1.786 \\
10 & 3.181 & 1.584 & 190.151 & 0.527 & 0.351 & 1.055 & 47.508 & 1.900 \\
11 & 3.193 & 1.589 & 190.749 & 0.529 & 0.353 & 1.059 & 47.657 & 1.906 \\
12 & 2.898 & 1.440 & 172.810 & 0.479 & 0.319 & 0.959 & 43.175 & 1.727 \\
13 & 3.040 & 1.514 & 181.779 & 0.504 & 0.336 & 1.009 & 45.416 & 1.816 \\
14 & 3.025 & 1.504 & 180.583 & 0.501 & 0.334 & 1.002 & 45.117 & 1.804 \\
15 & 3.122 & 1.554 & 186.563 & 0.517 & 0.345 & 1.035 & 46.611 & 1.864 \\
\hline
\end{tabular}

1) Result validation for Anechoic Chamber: Figure 9 displays the comparison of proposed consequences with the outcomes of smartphone sensors and applications. Figure 9a illustrates comparison of individual's velocity obtained from anechoic chamber. The error has been found approximately $3 \%$. Orientation of lower limb is also a important characteristic for human locomotion which, has been compared in Figure $9 \mathrm{~b}$ for anechoic environment. The errors found is negligible in this case. The traversed distance by an individual in this environment has been shown in Figure 9c The errors are approximately within $5 \%$.

\section{B. Results from Multipath Environment}

This section describes the results obtained from the real or normal environment, i.e. the laboratory room environment. To remain consistent, the same participants who participated in anechoic chamber scenario have been involved again. The waveforms back-scattered from shanks have been distinguished from other bio-mechanics activity in the same way as before. Figure 10 shows the frequency of lower leg movement attained from proposed STFT for the observation time of 2 seconds when the walking speed of participants was in fast, medium and of a slow pace. The figure illustrates the repetition of shank movements when the persons walk on the test bed. Figure 10a, 10c and 10e show the step frequency of female participants at different walking pace. Figure 10b, 10d, 


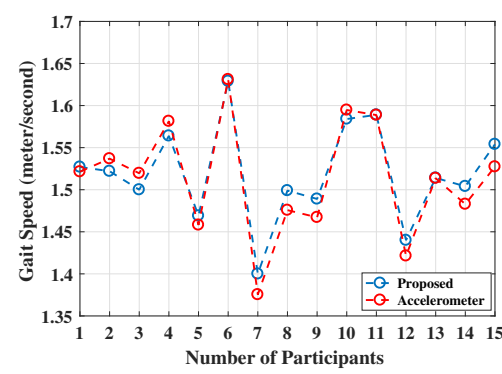

(a) Walking speed

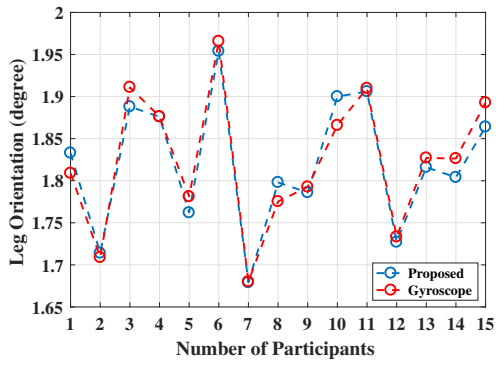

(b) Limb orientation

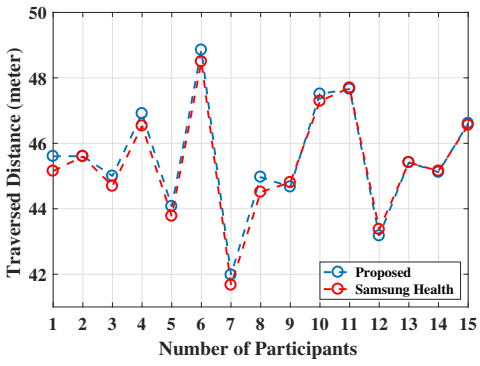

(c) Covered distance

Fig. 9: Commercial and UWB method comparison of gait parameters identified by proposed method and obtained from smartphone applications in ideal environment.

and 10f demonstrates the shank movement of males at different walking paces. The step frequency and walking speed are interrelated process thus, fast walk results in a greater number of steps and vice versa.
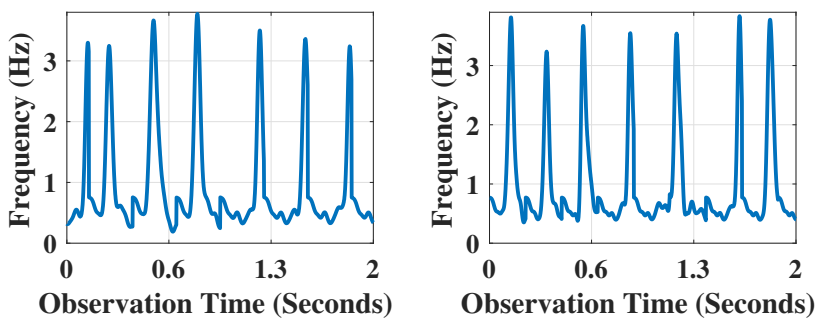

(a) Female walking at fast pace.

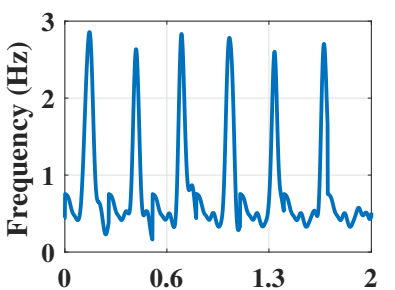

Observation Time (Seconds)

(b) Male walking at fast pace.

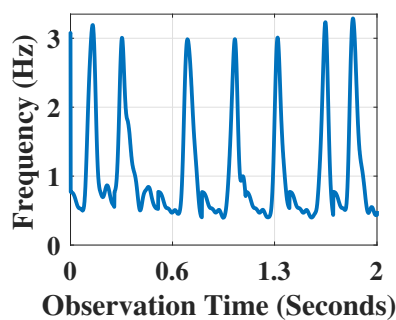

(c) Female walking at medium (d) Male walking at medium pace.
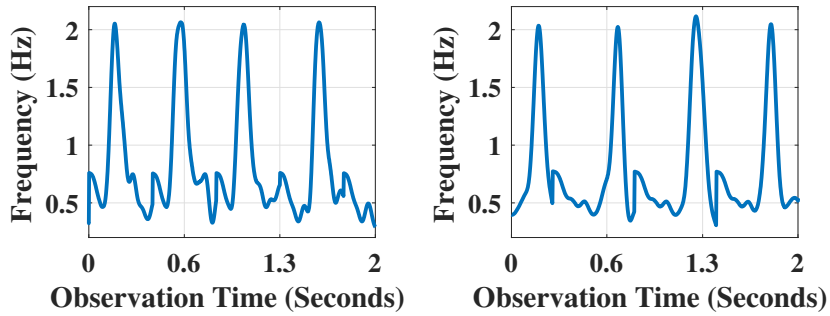

(e) Female walking at slow pace.

(f) Male walking at slow pace.

Fig. 10: Shank movement frequency of participants walking at different pace obtained through UWB radar in multi-path environment.

Therefore, the step frequency or the number of steps in one minute reaches up to $4 \mathrm{~Hz}$ (240 steps/minute) during fast walk and approximately $2 \mathrm{~Hz}$ (120 steps/minute) during the slow walk for both female and male. The discrepancy of step frequency for a person has been found when the participant reaches one end of test bed and walk slowly to take a turn to continue their walk. The step phase has been calculated (1/step frequency) from step frequency and detailed in Table IV] for all participants.
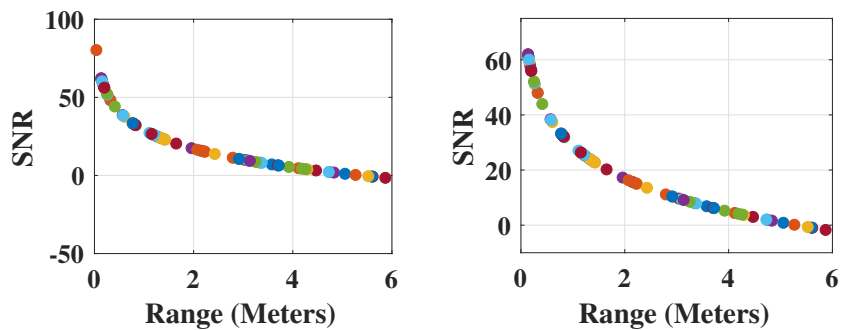

(a) $1^{\text {st }}$ female participant.

(b) $2^{\text {nd }}$ female participant.
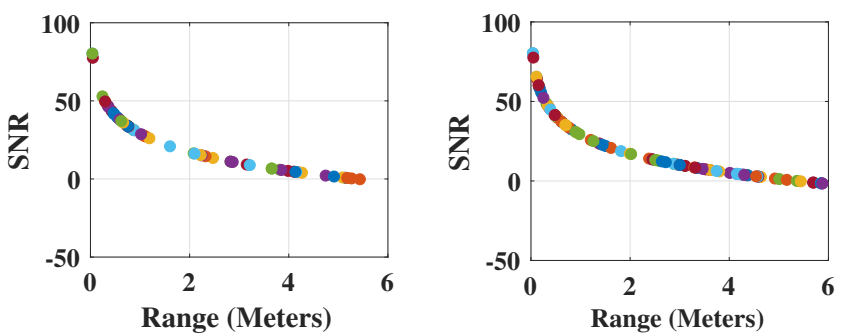

(c) $1^{\text {st }}$ male participant.

(d) $2^{\text {nd }}$ male participant.

Fig. 11: Relationship between SNR and range in multi-path environment during gait.

The relation between SNR and range in real environment is plotted in Figure 11 for two female and male participants. The noise figure is evaluated from the STP and SNR is measured from the scans with the help of detection information and logarithmic relation is found between SNR and range.

The route of participant's gait has been determined from Doppler effect like the experiment of anechoic chamber. The Doppler shift $f_{d}$ (described in Sectior II-E) has been measured during the observation time and plotted to check the route of a human walk in normal environment. Thus, the identified scans from shank have been plotted as received by time in Figure 12 The top and bottom of Figure $12 \mathrm{a}, 12 \mathrm{~b}, 12 \mathrm{c}$, and $12 \mathrm{~d}$ specify the nearest and furthest point from radar respectively. These figures illustrate the repetitive route of a participants walk. Table IV includes the average of parameters obtained from 




(a) $1^{\text {st }}$ female participant.

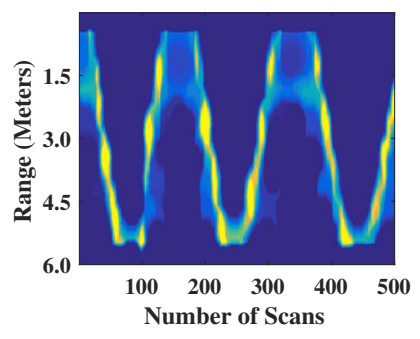

(c) $1^{\text {st }}$ male participant.

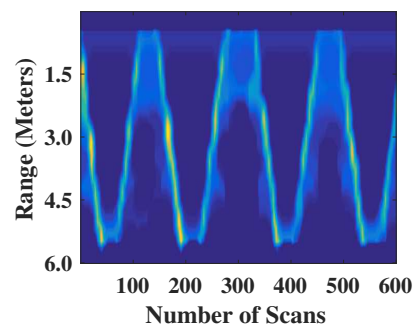

(b) $2^{\text {nd }}$ female participant.



(d) $2^{\text {nd }}$ male participant.

Fig. 12: Movement patterns on test bed in normal environment.

the experiment in normal or laboratory environment. The parameters of gait have been determined by following the same way in case of ideal environment.

TABLE IV: Results of gait analysis from multipath environment.

\begin{tabular}{lcccccccc}
\hline No & $\begin{array}{c}S_{f} \\
(\mathrm{~Hz})\end{array}$ & $\begin{array}{c}W_{s} \\
(\mathrm{~m} / \mathrm{s})\end{array}$ & $\begin{array}{c}C_{a} \\
(\mathrm{c} / \mathrm{min})\end{array}$ & $\begin{array}{c}S_{l} \\
(\mathrm{~m})\end{array}$ & $\begin{array}{c}S_{p} \\
(\mathrm{~s})\end{array}$ & $\begin{array}{c}S T_{l} \\
(\mathrm{~m})\end{array}$ & $\begin{array}{c}T_{d} \\
(\mathrm{~m})\end{array}$ & $\begin{array}{c}L_{o} \\
\left({ }^{0}\right)\end{array}$ \\
\hline & & & & & & & & \\
1 & 2.881 & 1.444 & 172.636 & 0.500 & 0.347 & 1.005 & 43.162 & 1.926 \\
2 & 3.033 & 1.520 & 181.898 & 0.497 & 0.329 & 0.993 & 45.447 & 2.933 \\
3 & 2.831 & 1.413 & 169.545 & 0.500 & 0.355 & 1.001 & 42.393 & 2.771 \\
4 & 3.176 & 1.582 & 190.268 & 0.561 & 0.398 & 1.123 & 47.572 & 3.108 \\
5 & 3.200 & 1.594 & 191.706 & 0.565 & 0.401 & 1.132 & 47.932 & 3.132 \\
6 & 2.946 & 1.467 & 176.507 & 0.521 & 0.369 & 1.042 & 44.132 & 2.883 \\
7 & 3.160 & 1.574 & 189.321 & 0.558 & 0.396 & 1.118 & 47.336 & 3.093 \\
8 & 3.147 & 1.568 & 188.542 & 0.556 & 0.394 & 1.113 & 47.141 & 3.080 \\
9 & 2.886 & 1.438 & 172.906 & 0.510 & 0.362 & 1.021 & 43.231 & 2.825 \\
10 & 2.863 & 1.426 & 171.523 & 0.506 & 0.359 & 1.013 & 42.885 & 2.802 \\
11 & 3.064 & 1.526 & 183.564 & 0.541 & 0.384 & 1.084 & 45.896 & 2.999 \\
12 & 3.308 & 1.648 & 198.218 & 0.585 & 0.415 & 1.170 & 49.560 & 3.238 \\
13 & 2.980 & 1.484 & 178.550 & 0.527 & 0.373 & 1.054 & 44.642 & 2.917 \\
14 & 3.110 & 1.54 & 186.326 & 0.550 & 0.390 & 1.100 & 46.587 & 3.044 \\
15 & 2.918 & 1.454 & 174.847 & 0.516 & 0.366 & 1.032 & 43.717 & 2.856 \\
& & & & & & & & \\
\hline
\end{tabular}

1) Result validation for multipath environment: Figure 13 shows the comparison of proposed outcomes with the outcomes of smartphone sensors and applications. Figure 13a demonstrate comparison of individual's velocity obtained from and normal environment respectively where the error has been found less than 3\%. Orientation of lower limb has been compared in Figure 13b. The traversed distance by an individual in normal environment has been presented in Figure $13 \mathrm{c}$ where the error is within $5 \%$.

\section{Discussion}

There are several human gait analysis techniques present in the literature aimed to parameters that identify and analyse

bipedal human gait. But, most of whether wearable and nonwearable techniques such as, sensors and cameras are included and had been employed with the help of expensive laboratory setting to identify and recover gait disorders. However, limited number of gait parameters had been obtained and could increase the number by including very function specific instruments which makes human subjects uncomfortable sometimes.

The work aims to propose an efficient, non-contact, and nonintrusive UWB gait identification technique by understanding of spherical trigonometry and radar principles heuristically. In this study a comprehensive framework has been developed to identify different types of person's walking pattern without hampering their privacy and comfort. A number of healthy human walking pattern involved for data accumulation in two type of environments (ideal and real). Due to the complexity of radar signals of human gait, a new theory has been developed. Further all the different walking style (male and female) towards and away from the radar has been evaluated. The rigorous analysis made this gait identification system robust and precise. Additionally, the obtained outcomes of this investigation have been tested and validated to prove this study which makes this method a powerful and productive human gait detection method that would be able to work even in harsh environments. This would open the door to clinical studies, security, health monitoring and many more perspectives.

\section{CONClusion \& Future WORK}

This paper presents the first ever description and experimental demonstration of a non-contact pulsed UWB sensor system to identify and extract human gait from other simultaneous bio-mechanic actions such as, arm swing, breathing and heart rates. A UWB radar sensor with in-house developed algorithms is used for data collection and processing. These signals are processed by STFT with fundamental radar principles employed to extract gait parameters including walking speed, step length, step phase, that define the quality and type of locomotion of a person. Also, important parameter such as distance travelled is evaluated which is effective for long term gait and mobility quality monitoring. The results show the effectiveness and advantages of pulsed UWB radar to analyse the quality of movement in an autonomous way. Research is currently underway to include - (i) stance (posture), joint angles, and duration of stops, (ii) leg/arm swing and stance times to understand/recognise abnormal gait patterns e.g., scissors, steppage, waddling, and propulsive gait.

\section{REFERENCES}

[1] K. Jordan, J. H. Challis, K. M. Newell, Walking speed influences on gait cycle variability, Gait \& posture 26 (1) (2007) 128-134.

[2] A. Muro-De-La-Herran, B. Garcia-Zapirain, A. Mendez-Zorrilla, Gait analysis methods: An overview of wearable and non-wearable systems, highlighting clinical applications, Sensors 14 (2) (2014) 3362-3394.

[3] S. Ghoussayni, et al., Assessment and validation of a simple automated method for the detection of gait events and intervals, Gait \& Posture 20 (3) (2004) 266-272.

[4] L. Wang, W. Hu, T. Tan, Recent developments in human motion analysis, Pattern recognition 36 (3) (2003) 585-601.

[5] W. Zang, Y. Li, Gait-cycle-driven transmission power control scheme for a wireless body area network, IEEE journal of biomedical and health informatics 22 (3) (2018) 697-706. 


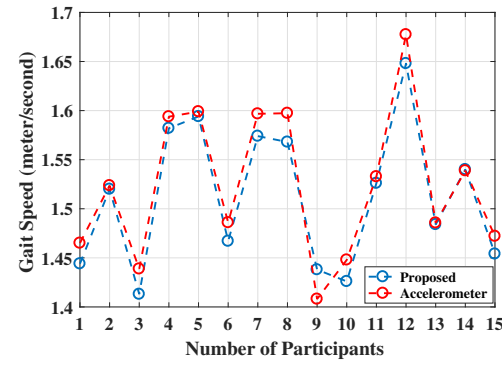

(a) Walking speed

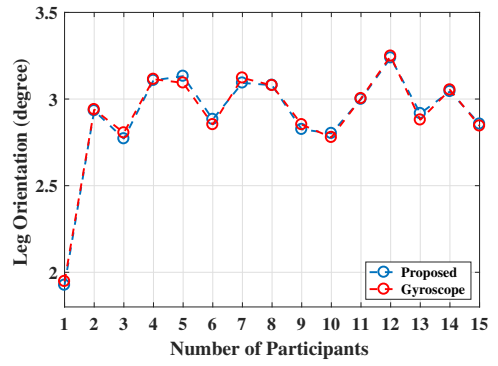

(b) Limb orientation



(c) Covered distance

Fig. 13: Commercial and UWB method comparison of gait parameters identified by proposed method and obtained from smartphone applications in multi-path environment.

[6] K. Watanabe, M. Hokari, Kinematical analysis and measurement of sports form, IEEE Transactions on Systems, Man, and Cybernetics-Part A: Systems and Humans 36 (3) (2006) 549-557.

[7] S. Kimmeskamp, E. M. Hennig, Heel to toe motion characteristics in parkinson patients during free walking, Clinical biomechanics 16 (9) (2001) 806-812.

[8] A. Salarian, et al., Ambulatory monitoring of physical activities in patients with parkinson's disease, IEEE Transactions on Biomedical Engineering 54 (12) (2007) 2296-2299.

[9] K. M. Tsiouris, D. Gatsios, G. Rigas, S. Konitsiotis, A. Antonini, D. I. Fotiadis, A decision support system based on rapid progression rules to enhance baseline evaluation of parkinson's disease patients, in: Biomedical \& Health Informatics (BHI), 2018 IEEE EMBS International Conference on, IEEE, 2018, pp. 329-332.

[10] J. Li, et al., Segmentation-based image copy-move forgery detection scheme, IEEE Transactions on Information Forensics and Security 10 (3) (2015) 507-518.

[11] D. Reynolds, Gaussian mixture models, Encyclopedia of biometrics (2015) 827-832.

[12] I. Rida, X. Jiang, G. L. Marcialis, Human body part selection by group lasso of motion for model-free gait recognition, IEEE Signal Processing Letters 23 (1) (2016) 154-158.

[13] S. Corazza, E. Gambaretto, L. Mundermann, T. P. Andriacchi, Automatic generation of a subject-specific model for accurate markerless motion capture and biomechanical applications, IEEE Transactions on Biomedical Engineering 57 (4) (2010) 806-812. doi:10.1109/ TBME. 2008.2002103

[14] D. A. Bruening, S. T. Ridge, Automated event detection algorithms in pathological gait, Gait \& posture 39 (1) (2014) 472-477.

[15] C. J. Payton, A. Burden, Biomechanical evaluation of movement in sport and exercise: the British Association of Sport and Exercise Sciences guide, Routledge, 2017.

[16] P. Ren, S. Hu, Z. Han, Q. Wang, S. Yao, Z. Gao, J. Jin, M. L. Bringas, D. Yao, B. Biswal, P. A. Valdes-Sosa, Movement symmetry assessment by bilateral motion data fusion, IEEE Transactions on Biomedical Engineering (2018) 1-1doi:10.1109/TBME.2018.2829749

[17] R. Williamson, B. J. Andrews, Gait event detection for fes using accelerometers and supervised machine learning, IEEE Transactions on Rehabilitation Engineering 8 (3) (2000) 312-319.

[18] P. Lopez-Meyer, G. D. Fulk, E. S. Sazonov, Automatic detection of temporal gait parameters in poststroke individuals, IEEE Transactions on Information Technology in Biomedicine 15 (4) (2011) 594-601.

[19] T. Everett, C. Kell, Human movement: an introductory text, Elsevier health sciences, 2010

[20] B. Najafi, K. Aminian, A. Paraschiv-Ionescu, F. Loew, C. J. Bula, P. Robert, Ambulatory system for human motion analysis using a kinematic sensor: monitoring of daily physical activity in the elderly, IEEE Transactions on Biomedical Engineering 50 (6) (2003) 711-723. doi:10.1109/TBME.2003.812189

[21] J. R. Rebula, et al., Measurement of foot placement and its variability with inertial sensors, Gait \& posture 38 (4) (2013) 974-980.

[22] B. R. Greene, et al., An adaptive gyroscope-based algorithm for temporal gait analysis, Medical \& biological engineering \& computing 48 (12) (2010) 1251-1260.

[23] A. Salarian, et al., Gait assessment in parkinson's disease: toward an ambulatory system for long-term monitoring, IEEE transactions on biomedical engineering 51 (8) (2004) 1434-1443.
[24] S. J. M. Bamberg, et al., Gait analysis using a shoe-integrated wireless sensor system, IEEE transactions on information technology in biomedicine 12 (4) (2008) 413-423.

[25] J. Bae, M. Tomizuka, A tele-monitoring system for gait rehabilitation with an inertial measurement unit and a shoe-type ground reaction force sensor, Mechatronics 23 (6) (2013) 646-651.

[26] E. Wentink, et al., Detection of the onset of gait initiation using kinematic sensors and emg in transfemoral amputees, Gait \& posture 39 (1) (2014) 391-396.

[27] Templo clinical gait analysis, https://www.contemplas.com/ clinical-gait-analysis.aspx

[28] Pressure mapping, force measurement $\&$ tactile sensors, https://www. tekscan.com/gait-analysis-systems

[29] D. Tahmoush, J. Silvious, Gait variations in human micro-doppler, International Journal of Electronics and Telecommunications 57 (1) (2011) 23-28.

[30] J. L. Geisheimer, W. S. Marshall, E. Greneker, A continuous-wave (cw) radar for gait analysis, in: Signals, Systems and Computers, 2001. Conference Record of the Thirty-Fifth Asilomar Conference on, Vol. 1, IEEE, 2001, pp. 834-838.

[31] G. Mokhtari, Q. Zhang, C. Hargrave, J. C. Ralston, Non-wearable uwb sensor for human identification in smart home, IEEE Sensors Journal 17 (11) (2017) 3332-3340. doi:10.1109/JSEN.2017.2694555

[32] J. Han, C. Nguyen, Development of a tunable multiband uwb radar sensor and its applications to subsurface sensing, IEEE Sensors Journal 7 (1) (2007) 51-58. doi:10.1109/JSEN.2006.888585

[33] Q. Liang, Radar sensor wireless channel modeling in foliage environment: Uwb versus narrowband, IEEE Sensors Journal 11 (6) (2011) 1448-1457. doi:10.1109/JSEN.2010.2097586

[34] S. P. Rana, et al., UWB localization employing supervised learning method, in: IEEE 17th International Conference on Ubiquitous Wireless Broadband (ICUWB), Salamanca, Spain, 2017.

[35] S. Rana, et al., Remote vital sign recognition through machine learning augmented UWB, in: IET 12th European Conference on Antennas and Propagation (EuCAP), London, United Kingdom, April, 2018.

[36] J. D. Taylor, Introduction to ultra-wideband radar systems, CRC press, 1994.

[37] F. C. Commission, In the matter of revision of part 15 of the commission's rules regarding ultra-wideband transmission systems, First Report And Order, ET Docket 98-153.

[38] M. A. Richards, Fundamentals of radar signal processing, Tata McGrawHill Education, 2005.

[39] J. Zhong, Y. Huang, Time-frequency representation based on an adaptive short-time fourier transform, IEEE Transactions on Signal Processing 58 (10) (2010) 5118-5128. 